\title{
LA AUTOESTIMA y LOS VALORES ORGANIZACIONALES EN ESTUDIANTES UNIVERSITARIOS DE LIMA
}

\author{
Alejandro Loli Pineda \\ Ernestina López Vega \\ María Atalaya Pisco
}

\begin{abstract}
RESUMEN
La autoestima y los valores organizacionales en estudiantes de la universidad pública y privada de Lima, es un estudio desarrollado en una muestra de 1464 sujetos de una Universidad Pública y una Universidad Privada de las más antiguas tradicionales del país, con el objeto de conocer la jerarquía de valores organizacionales percibidos por los jóvenes universitarios que esperan de la organización en la que desearían trabajar a la culminación de sus estudios; la relación entre la autoestima y ciertos rasgos demográficos, y también las diferencias entre los valores organizacionales y dichos rasgos demográficos. Los resultados indican que los estudiantes universitarios le dan mayor importancia a la limpieza, servicio al cliente, y a la calidad del producto y del servicio, y menor importancia a la honradez, a la responsabilidad y al respeto a la persona. Igualmente, se encuentra correlación positiva entre la autoestima personal y el valor limpieza, negativa entre la autoestima y el valor responsabilidad y respeto a la persona. El análisis cruzado de los niveles de autoestima por universidades nos indica que no existe diferencia estadística significativa entre la Universidad Pública y Privada, no obstante hay predominio de la autoestima baja tanto en la primera como en la segunda, salvo cuando interviene el género donde la diferencia es significativa con predominio de la autoestima baja en varones y media en mujeres.
\end{abstract}

Palabras Clave: Autoestima, valores organizacionales, estudiantes universitarios.

\begin{abstract}
The selfesteem and the organizational values in students of the public and private university of Lima, it is a study developed in a sample of 1464 subject of a Public University and a Private University of the oldest and traditional of the country, in order to knowing the hierarchy of values organizational perceived by the university youths that expect from the organization in which you they would want to work to the culmination of their studies; the relationship between the selfesteem and certain demographic features, and also the differences among the values organizational and this demographic features. The results indicate that the university students give bigger importance to the cleaning, service to the client, and to the quality of the product and of the service, and smaller importance to the honesty, to the responsibility and the respect to the person. Equally, he/she is positive correlation between the personal selfesteem and the value cleaning, negative between the selfesteem and the value responsibility and respect to the person. The crossed analysis of the levels of self-esteem for universities indicates us that difference significant statistic doesn't exist among the Public University and Deprived nevertheless there is prevalence of the low selfesteem as much as in the first one as in second o' clock, except for when the gender intervenes where the difference is significant with prevalence of the low selfesteem in mens and mediates in women.
\end{abstract}

Key words: selfesteem, organizational values, university students.

Los autores agradecen el apoyo de la psicóloga Clemencia Villegas Ortiz y los alumnos Eliana Zubiaga Atoche, Jimmy Mallqui Bacilio, Yovana Huamán Bustamante, Marcelino De la Cruz Rodríguez, Lenín Barreta Cáceres y Gianina Hurtado Paredes. 


\section{INTRODUCCIÓN}

En los últimos años el país ha experimentado cambios importantes en la vida económica, política, social y cultural, a cuyos cambios ha contribuido el desarrollo tecnológico, permitiendo una percepción diferente del mundo.

Las organizaciones empresariales, entre otras, probablemente estén aprendiendo a más velocidad las exigencias de la globalización a fin de competir en mejores condiciones en el mercado nacional e internacional Los estudios desarrollados sobre el tema (T Peters y R.H. Waterman, 1982; R. Curvin, 1989; J. Cruz, 1996; M. Rodriguez, 1993), nos informan sobre la importancia de la autoestima y los valores organizacionales en el éxito personal y organizacional. En 1998, A. Loli; E. López y M. Atalaya realizan una investigación sobre el tema en 105 Gerentes de Microempresas del Cono Este de Lima, encontrando correlación significativa entre la autoestima y algunos valores organizacionales (lealtad, amor al trabajo, trabajo en equipo) y otras variables demográficas. Igualmente, el estudio presenta una jerarquía de valores organizacionales calificados por la misma muestra, confirmando de este modo el rol protagónico que tienen tanto la autoestima como los valores en las organizaciones.

Si lo señalado ocurre en el presente, la necesidad de afrontar los retos personales y sociales en el futuro, corresponden con mayor propiedad a los niños y a los jóvenes. Por ello nuestra preocupación está encaminada a conocer la jerarquía de valores organizacionales que los jóvenes aspiran encontrar en el ejercicio de su profesión en las organizaciones laborales a la culminación de sus estudios; la relación entre la autoestima personal y los valores organizacionales: y las diferencias de la autoestima y los valores organizacionales con variables demográficas, tales como la edad, género, procedencia, ciclo de estudios, etc.

\section{METODOLOGIA}

La metodología empleada es la de Ranking (medida de variabilidad) para determinar la jerarquía de valores organizacionales; la correlación de Pearson se empleó para determinar la asociación entre la autoestima y los valores organizacionales; el puntaje " $t$ " de Student para determinar las diferencias de la autoestima con las variables demográficas, y los valores con las mismas variables; en cambio para ver los niveles de autoestima en relación con dichas variables se utilizó el análisis cruzado mediante el Chi Cuadrado.

\section{LA MUESTRA}

La muestra estuvo conformada por 1,464 estudiantes universitarios de dos universidades más grandes y tradicionales del Perú (una Pública y la otra Privada) que realizan estudios de distintas carreras profesionales. Fue seleccionada de manera intencional de acuerdo con la disposición de cada universidad (Pública y Privada); es decir, tenían que disponer de las mismas carreras profesionales y con un mínimo de diez estudiantes por carrera en cada Universidad. En el caso de la Universidad Pública, la muestra fue de 764 estudiantes que representa el 3\% de su población total; en el Caso de la Universidad Privada; la muestra fue de 700 estudiantes que representa el $5 \%$ de su población total. 


\section{TECNICA DE RECOLECCION DE DATOS}

La técnica empleada para la recolección de la información fue la administración colectiva en los salones dc clase de las universidades que formaron parte de la presente muestra, lograda a través de egresados de psicología que fueron especialmente entrenados para la administración del Inventario de Autoestima y la Lista de Valores Organizacionales.

\section{INSTRUMENTO}

Los instrumentos que sirvieron de filtro para la recolección de la información fueron el Inventario de Autoestima (ALPEL), Forma-AD y la Lista de Valores Organizacionales (ALPEL), Forma-VA, ambos instrumentos construidos para una investigación similar con gerentes de microempresas en 1998 (A. Loli y Col., 1998).

El Inventario de Autoestima es una prueba de 46 itemes; producto de un análisis de contenido de 120 estímulos, que sometido a juicio de los jueces o expertos se determinó la consistencia interna de la prueba. El instrumento incorpora información sobre cinco factores (conocimiento, concepto, respeto, evaluación y valoración de sí mismo), que teóricamente se afirma, son los elementos base sobre los cuales se construye el concepto de autoestima. En el momento actual se viene trabajando en la ampliación de la muestra para darle un tratamiento estadístico de validez y confiabilidad. La Lista de Valores Organizacionales, en número de 12, se determinó luego de que se aplicó una lista de 36 valores relacionados a las organizaciones, a una muestra de 105 gerentes de microempresas, solicitándoles que establecieran una jerarquía de valores en orden de importancia.

\section{RESULTADOS}

\section{Jerarquización de los valores organizacionales}

El cuadro $\mathrm{N}^{\circ} 01$ nos indica el orden de los promedios con que han sido evaluados los 12 valores organizacionales. Cuando se analiza la muestra total, se encuentra que el valor "limpieza" (8.98) es el más importante seguido por "servicio al cliente" (8.91) y "calidad del producto o servicio" (7.67); mientras que "honradez "( 4.97). "responsabilidad" (3.87) y "respeto a la persona" (3.59) son los menos importantes.

Todo lo cual significa que se sacrifica la honradez, la responsabilidad y el respeto a la persona por un mejor servicio al cliente, una mejor limpieza y mejor calidad del producto o del servicio. Además, parece que la posición de estudiar en una u otra universidad no influye en esta percepción.

- Entre el cuadro $\mathrm{N}^{\circ} 2$ y 12 (Ver cuadros en documento original) se analizan la jerarquía de valores organizacionales en relación con variables demográficas, encontrando que la estructura jerárquica resultante de la muestra total es una constante; y las diferencias encontradas mediante el puntaje " $\mathrm{t}$ " de student nos indican que, mientras los estudiantes de la Universidad Pública relevan valores centrados en la persona y su relación con la sociedad, los de la Universidad Privada centran su atención en valores instrumentales y prácticos.

\section{La Autoestima y los valores organizacionales}

El cuadro $\mathrm{N}^{\circ} 13$ analiza la correlación entre la autoestima y los valores 
organizacionales. El análisis de la muestra total nos indica que existen asociaciones estadísticas significativas entre autoestima y los valores de limpieza $(r=.115)$, responsabilidad $(\mathrm{r}=-.123)$ respeto a la persona $(\mathrm{r}=-.052)$, notándose que en todos los casos las correlaciones son bajas, y en limpieza es directa, mientras que en los otros dos son inversos. Esto nos indica que, a mayor autoestima hay mayor tendencia a valorar la limpieza, y una menor valoración de la responsabilidad y un menor respeto a la persona.

Entre el cuadro 14 y 30 (Ver cuadros en documento original) se analizan detalles de la correlación entre la autoestima y los valores organizacionales en relación a variables demográficas, encontrando resultados similares a la correlación del cuadro $\mathrm{N}^{\circ} 13$. La comparación con las variables demográficas no arroja diferencia estadística significativa, salvo con la intervención de la variable género, donde hay diferencia significativa, siendo más alta en mujeres (36.23) que en varones (36.00), probablemente por influencia de la cultura y la familia, donde la mujer aprende a respetarse y valorarse más pronto que los varones y parece persistir en el tiempo porque la intervención de la edad no influye en nada.

\section{Los niveles de Autoestima}

Los cuadros que van del $\mathrm{N}^{\circ} 31$ al $\mathrm{N}^{\circ} 35$ nos muestran los niveles de autoestima por universidades y también por variables demográficas, en ellos encontramos que no existen diferencias estadísticas significativas, salvo cuando interviene la variable género, donde sí hay diferencia estadística significativa (.006) entre varones y mujeres. con predominio de la autoestima baja (39.9) en estudiantes universitarios varones y autoestima media (36.00) con estudiantes universitarias mujeres.

\section{DISCUSIÓN}

La autoestima como los valores surgen en el hombre y los acompañan a lo largo de su existencia, permitiéndole actuar y transmitir a través de sus actitudes y comportamiento para influir en la formación de rasgos psíquicos en las personas, grupos y organizaciones con los cuales se relaciona e interactúa. Los resultados de la investigación nos permite confirmar la relación entre la autoestima y los valores organizacionales.

La jerarquía de valores que toda persona posee se constituye en su sistema de valores y este " ... sistema se identifica en razón de la importancia que se conceda a los valores ... " (Robbinson, 1994). Este sistema de valores es el que trasmiten los fundadores y directivos a sus organizaciones y cada empleado que se incorpora a ella transmite los suyos a la organización, llegándose a un proceso de ajuste mutuo de influencias para constituir el sistema de valores de la organización.

Es posible que en los resultados que se alcanza en esta investigación, los integrantes de la muestra hayan expresado su percepción sobre la base de su propio sistema de valores, pero es interesante ver cómo la puntualidad que resultaba de vital importancia para los 105 gerentes de las microempresas investigadas por A. Loli y colaboradores (1998), en esta muestra es desplazada al cuarto lugar en importancia dándole prioridad a la limpieza, el servicio al cliente y la calidad del producto o servicio; es más, con este objeto se sacrifica la honradez, la responsabilidad." el respeto a la persona. que se sitúan en los últimos lugares en importancia. situación bastante peligrosa que las instituciones deben advertir y prepararse para encarar los efectos que la política económica actual está ocasionando en la 
percepción de los jóvenes, más todavía si se toma en cuenta que los valores tienen un gran impacto en el desempeño de las organizaciones (Korrer y Heskett. en J. Stoner y otros, 1996), o es que para los jóvenes universitarios los valores " ... representan la convicción básica de que una forma de conducta específica o de condición última de la vida son preferibles, en términos personales o sociales a otra forma de conducta ... contrarias u opuestas ... " como afirma M. Rokeach (1973). Aunque cuando se separa la muestra en estudiantes de Universidad Pública y Privada, se encuentran diferencias estadísticas significativas en puntualidad y limpieza, siendo más importantes para estudiantes de la Universidad Privada que Pública, pero también existe diferencia estadística significativa en confianza, respeto a la persona y honradez, siendo más importante para estudiantes de la Universidad Pública.

Por otro lado, se afirma que es innegable que el " ... punto de partida en la inductiva de los valores (positivos) ... sea el incremento de la autoestima ..." (G. Mora, 1996), o es que la autoestima es el resultado que tiene el individuo de la "internalización de las normas y los valores del grupo social y de su adecuado desempeño." (Sherif y Sherif, 1996); por encima de la disonancia, la autoestima juega un papel modulador en la relación entre el clima organizacional y la satisfacción con el trabajo'" (J. De la Poza y J. Prior. 1988: Mossholder, 1981; Adler. 1980). de manera que " ... el conflicto de función tiene relación negativa con la satisfacción laboral, el desempeño. el compromiso organizacional ." la participación que da autoestima...."; es decir, cuanto mayor es cl conflicto de función menor será la participación que da autoestima. Lo cierto es que la autoestima reforzada es capaz de elevar la productividad e ir mucho más allá para mejorar su actitud en el servicio, elevando además su autoestima y el cambio personal hasta sentirse orgulloso, satisfecho con su empleo. "Este cambio de percepción laboral sólo es posible, como establece Yerkes (1978), con el desarrollo de la autoestima hasta el nivel que Maslow (1952) señala como nivel de autorrealización personal, que nosotros llamamos nivel de servicio" (Cruz, 1997).

De acuerdo con nuestros resultados, independientemente a la prevalencia de la autoestima o de los valores en el éxito de las instituciones, hallamos que existe correlación estadística significativa entre la autoestima y los valores organizacionales, siendo positiva en limpieza, y negativa en responsabilidad y respeto a la persona; en otros términos, cuanto mayor es la autoestima mayor es el valor que se le otorga a la limpieza, y menor la valoración a la responsabilidad y el respeto a la persona. Parece que estamos frente a una situación innegable en que el exceso de autoestima personal conduce a presumir y desalentar su responsabilidad organizacional y también el respeto a las personas. Entonces, no se debe hablar sólo de autoestima positiva o negativa, alta o baja sino que existe un máximo más allá del cual la autoestima es perjudicial. situación en la cual. la conducta personal como organizacional son anómalas. donde sólo la rectitud de valores sólidos sería capaz de controlar. Podría creerse que el cambio de percepción laboral mejore cuando los estudiantes de la muestra concluyan con esta etapa de formación y busquen la autorrealización profesional en la vida real, como señala Yerkes (en Cruz. 1997), pero no, hay un grupo de estudiantes que trabajan y estudian, especialmente aquellos que sobrepasan los 21 años, donde la correlación es similar.

Cuando la muestra es separada en Universidad Pública y Privada, no varía mucho, se incrementa a lo ya señalado, la correlación de la autoestima con puntualidad de manera positiva, y con trabajo en equipo en forma negativa. Es decir, a mayor autoestima de los estudiantes de la Universidad Pública mayor valoración a la puntualidad y menor valoración al trabajo en equipo, resultado que es coherente con lo encontrado por A. Loli y 
colaboradores (1998) en una muestra de gerentes de microempresas; mientras que en la Universidad Privada sólo existe asociación significativa y negativa con responsabilidad; o sea, a mayor autoestima menor valoración a la responsabilidad. Probablemente se debe a que la expectativa de los estudiantes es esencialmente de dirección, pero entendido en el sentido tradicional, donde los equipos de trabajo no tenían lugar.

Ahora bien, el análisis cruzado de los niveles de autoestima con algunos rasgos demográficos (procedencia, edad, estado civil) no correlaciona, salvo con género donde la asociación es estadísticamente significativa. predominando la autoestima baja en varones y autoestima media en mujeres. permitiéndonos inferir que la influencia familiar más que la cultural es dominante en el desarrollo de la autoestima, persistiendo incluso en esta etapa de la formación profesional. Por consiguiente. parece indiscutible reforzar la autoestima tanto en varones como en mujeres con el objeto de elevar la productividad e ir mucho más allá para mejorar su actitud en el servicio (Cruz, 1997) o en el producto cuando concluyan sus estudios.

\section{CONCLUSIONES}

Basado en los resultados del análisis estadístico de la investigación se puede arribar a las siguientes conclusiones:

1. La escala de Valores Organizacionales que los estudiantes universitarios esperan encontrar al concluir sus estudios, como los más importantes (de más a menos importante) son: limpieza, servicio al cliente, calidad del producto o del servicio, puntualidad, trabajo en equipo, disciplina, amor al trabajo, confianza, deseo de superación, honradez, responsabilidad y respeto a la persona. Es decir, desde la perspectiva de los futuros profesionales se debe sacrificar la honradez, la responsabilidad y el respeto a la persona por mantener la limpieza, el servicio al cliente y la calidad del producto o servicio.

2. La comparación de la escala de Valores Organizacionales entre la Universidad Pública y Privada, nos muestra diferencias significativas en puntualidad y limpieza, siendo mayor en la Universidad Privada que en la Pública. También hay diferencia significativa en confianza, respeto a la persona y honradez, siendo mayor en la Universidad Pública que en la Privada. Vale decir, que mientras los estudiantes de la Universidad Privada se centran en valores instrumentales, aquellos de la Universidad Pública centran su atención en valores vinculados a la persona.

3. La correlación entre la Autoestima y los Valores Organizacionales nos indica que existe asociación estadística significativa y positiva con limpieza y negativa con responsabilidad y respeto a la persona. Es decir, a mayor autoestima mayor la tendencia a valorar la limpieza, y por otro lado, a mayor autoestima menor valoración de la responsabilidad y menor respeto a la persona. De cuyos resultados se puede inferir que, cuando la autoestima es alta, crece el "ego" en exceso, hay una mayor preocupación por mantenerla (mediante la limpieza y la pulcritud), disminuye su responsabilidad organizacional, también merma el respeto a la persona; en su caso, puede incluso hasta exigir y no practicarlo. 
4. Un análisis cruzado de los niveles de autoestima por universidades indica que no existe diferencia estadística significativa; sin embargo, la tendencia predominante es la autoestima baja, tanto en la Universidad Pública como en la Privada. En cambio, cuando este análisis se realiza tomando en cuenta el género, hay diferencia estadística significativa con predominio de autoestima baja en varones y autoestima media en mujeres. Este resultado nos estaría indicando que hay mayor aceptación, mejor concepto, mayor conocimiento, mayor valoración de sí mismo en las mujeres que en los varones. Por lo demás, el análisis cruzado de los niveles de autoestima con los otros rasgos demográficos no arroja diferencia estadística importante.

\section{BIBLIOGRAFIA}

ASPE, Virginia; LOPEZ, Ana. Hacia un desarrollo humano: Valores, actitudes y hábitos. México, Edit. Limusa, 1999.

BONET, José. Autoestima. España, Edit. Sal Terrae, 1994.

CLEMES, Harris y BEAN, Reynold. Cómo desarrollar la autoestima en los niños. España, Edit. Debate.S.A., 1996.

CRUZ RAMIREZ, José. Autoestima y gestión de la calidad. México, Edit. Iberoamericana, 1997.

HUNTER, Lewis. La cuestión de los valores humanos. España, Barcelona, Edit. Gedisa, 1998.

LEBOW, Rob. La clave de la productividad empresarial. México, Panorama Editorial S.A. 1995.

LOLI, Alejandro; LOPEZ, Ernestina. Autoestima y valores en la calidad y la excelencia. Lima, Perú, Instituto de Investigaciones Psicológicas, UNMSM, 1997.

LOLI, Alejandro; LOPEZ, Ernestina; ATALAYA, María. La autoestima y los valores en las empresas de Lima. Perú, Instituto de Investigaciones Psicológicas, UNMSM, 1998.

MEYERS, David G. Psicología social. México, Edit. McGraw Hill. 1995.

MORA. Guillermo; SARMIENTO, Enma:OSSA. John. Valores humanos y actitudes positivas. Colombia, McGraw Hill. 1995.

O'CONNOR, Michael. Administración por valores. Colombia, Edit. Norma, 1997.

ONATE. M.P. El Autoconcepto,formación, medida e implicaciones en la personalidad. Madrid, Edit. Narcea, 1989.

ROBBINS, Stephen. Comportamiento organizacional. México, Edit. Prentice Hall Hispanoamericana, 1994.

RODRIGUEZ, Mauro. Los valores, clave de la excelencia. México, Edit. McGraw HiI, 
1993.

STONER, James; FREEMAN, Edward; GUILBET, Daniel. Administración. México, Edil. Prentice Hall Hispanoamericana, 1996.

THEVENET, Maurice. Auditoría de la cultura empresarial. España, Madrid, Edit. Diaz de Santos, 1992. 


\section{CUADRO $\mathbf{N}^{\circ} 1$ \\ JERARQUIZACIÓN DE LOS VALORES ORGANIZACIONALES POR \\ UNIVERSITARIOS Y MUESTRA TOTAL}

\begin{tabular}{|c|c|c|c|c|c|c|c|c|c|}
\hline $\mathbf{N}^{\mathbf{o}}$ & $\begin{array}{r}\text { UNIVERSIDA } \\
(\mathbf{N}=?\end{array}$ & PUB & & $\begin{array}{r}\text { UNIVERSID } \\
\qquad(\mathrm{N}=\end{array}$ & $\begin{array}{l}\text { D PR } \\
\text { 00) }\end{array}$ & DA & $\begin{array}{r}\text { MUESTRA } \\
(\mathrm{N}=14\end{array}$ & $\begin{array}{l}\text { TOTA } \\
\text { 64) }\end{array}$ & \\
\hline ORD. & VALORES & $\mathbf{M}$ & D.S & VALORES & $\mathbf{M}$ & D.S. & VALORES & $\mathbf{M}$ & D.S. \\
\hline 1 & Servicios al cliente & 8.91 & 2.98 & Limpieza & 9.18 & 2.80 & Limpieza & 8.98 & 2.92 \\
\hline 2 & Limpieza & 8.8 & 3.02 & $\begin{array}{l}\text { Servicios al } \\
\text { cliente }\end{array}$ & 8.91 & 2.81 & $\begin{array}{l}\text { Servicios al } \\
\text { cliente }\end{array}$ & 8.91 & 2.9 \\
\hline 3 & Calidad & 7.8 & 3.42 & Puntualidad & 7.66 & 3.14 & Calidad & 7.67 & 3.4 \\
\hline 4 & Trabajo en Equipo & 7.14 & 3.23 & Calidad & 7.53 & 3.37 & Puntualidad & 7.29 & 3.24 \\
\hline 5 & Puntualidad & 6.96 & 3.30 & $\begin{array}{l}\text { Trabajo en } \\
\text { Equipo }\end{array}$ & 7.27 & 3.15 & $\begin{array}{l}\text { Trabajo en } \\
\text { Equipo }\end{array}$ & 7.20 & 3.19 \\
\hline 6 & Disciplina & 6.65 & 3.13 & Amor al Trabajo & 6.65 & 3.26 & Disciplina & 6.63 & 3.09 \\
\hline 7 & Confianza & 6.59 & 4.04 & Disciplina & 6.61 & 3.04 & $\begin{array}{l}\text { Amor al } \\
\text { Trabajo }\end{array}$ & 6.53 & 3.27 \\
\hline 8 & Amor al Trabajo & 6.42 & 3.28 & Confianza & 6.16 & 3.00 & Confianza & 6.38 & 3.03 \\
\hline 9 & Superación & 5.84 & 3.07 & Superación & 6.05 & 2.98 & Superación & 5.94 & 3.03 \\
\hline 10 & Honradez & 5.17 & 2.95 & Honradez & 4.76 & 2.86 & Honradez & 4.97 & 2.91 \\
\hline 11 & Responsabilidad & 3.9 & 2.36 & Responsabilidad & 3.84 & 2.36 & Responsabilidad & 3.87 & 2.36 \\
\hline 12 & $\begin{array}{l}\text { Respeto a la } \\
\text { Persona }\end{array}$ & 3.81 & 3.06 & $\begin{array}{l}\text { Respeto a la } \\
\text { Persona }\end{array}$ & 3.36 & 2.94 & $\begin{array}{l}\text { Respeto a la } \\
\text { Persona }\end{array}$ & 3.59 & 3.01 \\
\hline
\end{tabular}




\section{CUADRO $\mathrm{N}^{\circ} 2$}

\section{COMPARACIÓN DE LOS VALORES ORGANIZACIONALES POR UNIVERSIDADES}

\begin{tabular}{|c|c|c|c|c|c|c|}
\hline \multirow{2}{*}{\begin{tabular}{|l|} 
UNIVERSIDADES \\
Valores Organizacionales
\end{tabular}} & \multicolumn{2}{|c|}{$\begin{array}{rr}\text { PUBLICA } & \mathrm{N}= \\
764 & \end{array}$} & \multicolumn{2}{|c|}{$\begin{array}{rr}\text { PRIVADA } & \mathbf{N}= \\
700 & \end{array}$} & \multirow[t]{2}{*}{$\mathbf{t}$} & \multirow[t]{2}{*}{ Sign. } \\
\hline & $\mathbf{M}$ & D.S & $\mathbf{M}$ & D.S. & & \\
\hline Puntualidad & 6.96 & 3.3 & 7.66 & 3.14 & -4.16 &, $000 *$ \\
\hline Calidad del Producto y Servicio & 7.88 & 3.42 & 7.53 & 3.37 & 1.51 & 0.131 \\
\hline Limpieza & 8.8 & 3.02 & 9.18 & 2.80 & -2.52 &, $012^{*}$ \\
\hline Deseo de Supervisión & 5.84 & 3.07 & 6.05 & 2.98 & -1.32 & 0.186 \\
\hline Amor al Trabajo & 6.42 & 3.28 & 6.65 & 3.26 & -1.35 & 0.177 \\
\hline Confianza & 6.59 & 3.04 & 6.16 & 2.99 & 2.72 &, $007 *$ \\
\hline Responsabilidad & 3.9 & 2.36 & 3.84 & 2.36 & 0.47 & 0.64 \\
\hline Respeto a la Persona & 3.8 & 3.06 & 3.36 & 2.94 & 2.84 & 0.005 \\
\hline Trabajo en Equipo & 7.14 & 3.24 & 7.27 & 3.15 & -0.74 & 0.461 \\
\hline Honradez & 5.17 & 2 & 4.76 & 2.86 & 2.71 &, $007 *$ \\
\hline Servicio al Cliente & 8.91 & 2.98 & 8.91 & 2.81 & -0.01 & 0.99 \\
\hline Disciplina & 6.65 & 3.13 & 6.61 & 3.04 & 0.26 & 0.795 \\
\hline
\end{tabular}

CUADRO No 3

JERARQUIZACIÓN DE LOS VALORES ORGANIZACIONALES SEGÚN PROCEDENCIA (MUESTRA TOTAL)

\begin{tabular}{|c|c|c|c|c|c|}
\hline \multirow[b]{2}{*}{ Valores Organizacio. } & \multicolumn{2}{|c|}{$\begin{array}{c}\text { LIMA } \\
\text { No }^{\circ} 1236\end{array}$} & \multirow[b]{2}{*}{ Valores Organizacio. } & \multicolumn{2}{|c|}{$\begin{array}{c}\text { PRIVADA } \\
N^{0} 228\end{array}$} \\
\hline & $\mathrm{M}$ & D.S. & & $\mathrm{M}$ & D.S. \\
\hline Limpieza & 8.96 & 2.91 & Limpieza & 9.07 & 2.97 \\
\hline Servicio al Cliente & 8.94 & 2.91 & Servicio al Cliente & 8.79 & 2.82 \\
\hline Calidad Prod. y Servic. & 7.66 & 3.4 & Calidad Prod. y Servic. & 7.73 & 3.39 \\
\hline Puntualidad & 7.37 & 3.22 & Trabajo en Equipo & 7.18 & 3.31 \\
\hline Trabajo en equipo & 7.21 & 3.17 & Puntualidad & 6.86 & 3.35 \\
\hline Disciplina & 6.67 & 3.09 & Confianza & 6.66 & 2.97 \\
\hline Amor al Trabajo & 6.52 & 3.27 & Amor al Trabajo & 6.58 & 3.29 \\
\hline Confianza & 6.33 & 3.04 & Disciplina & 6.43 & 3.06 \\
\hline Deseo de Superación & 5.94 & 3.01 & Deseo de Superación & 5.95 & 3.11 \\
\hline Honradez & 4.94 & 2.91 & Honradez & 5.13 & 2.93 \\
\hline Responsabilidad & 3.91 & 2.37 & Responsabilidad & 4.02 & 3.21 \\
\hline Respeto & 3.52 & 2.97 & Respeto & 3.69 & 2.28 \\
\hline
\end{tabular}


DE EDAD (MUESTRA TOTAL)

\begin{tabular}{|l|c|c|l|c|c|}
\hline \multicolumn{3}{|c|}{$\begin{array}{c}\text { Edad: 16-20 } \\
\mathbf{N}^{\mathbf{0}} \mathbf{6 6 8}\end{array}$} & & \multicolumn{2}{c|}{$\begin{array}{c}\text { Edad: 21-48 } \\
\mathbf{7 9 6}\end{array}$} \\
\hline Valores Organizacio. & $\mathrm{M}$ & D.S. & Valores Organizacio. & M & D.S. \\
\hline Servicio al Cliente & 9 & 2.88 & Limpieza & 9.05 & 2.91 \\
\hline Limpieza & 8.9 & 2.94 & Servicio al Cliente & 8.84 & 2.91 \\
\hline Calidad Prod. y Servic. & 7.72 & 3.37 & Calidad Prod. y Servic. & 7.62 & 3.42 \\
\hline Trabajo en equipo & 7.32 & 3.16 & Puntualidad & 7.3 & 3.28 \\
\hline Puntualidad & 7.28 & 3.2 & Trabajo en equipo & 6.3 & 3.22 \\
\hline Disciplina & 6.62 & 3.03 & Amor al Trabajo & 6.22 & 3.16 \\
\hline Confianza & 6.58 & 3.06 & Disciplina & 6.64 & 3.13 \\
\hline Amor al Trabajo & 6.35 & 3.39 & Confianza & 6.22 & 2.99 \\
\hline Deseo de Superación & 5.82 & 3.02 & Deseo de Superación & 6.04 & 3.03 \\
\hline Honradez & 5.03 & 2.93 & Honradez & 4.92 & 2.9 \\
\hline Responsabilidad & 3.83 & 2.32 & Responsabilidad & 3.91 & 2.39 \\
\hline Respeto & 3.56 & 3 & Respeto & 3.62 & 3.02 \\
\hline
\end{tabular}

\section{CUADRO $\mathrm{N}^{\circ} 9$ \\ JERARQUIZACIÓN DE LOS VALORES ORGANIZACIONALES SEGÚN GENERO (MUESTRA TOTAL)}

\begin{tabular}{|l|c|c|l|c|c|}
\hline \multicolumn{3}{|c|}{$\begin{array}{c}\text { MASCULINO } \\
\mathbf{N}^{\mathbf{0}} \mathbf{7 3 6}\end{array}$} & & \multicolumn{2}{c|}{$\begin{array}{c}\text { FEMENINO } \\
\mathbf{N}^{\mathbf{0}} \mathbf{7 2 8}\end{array}$} \\
\hline Valores Organizacion. & $\mathbf{M}$ & D.S. & Valores Organizacio. & M & D.S. \\
\hline Servicio al Cliente & 8.89 & 2.93 & Limpieza & 9.29 & 2.78 \\
\hline Limpieza & 8.67 & 3.03 & Servicio al Cliente & 8.94 & 2.87 \\
\hline Calidad Prod. y Servic. & 7.53 & 3.47 & Calidad Prod. y Servic. & 7.8 & 3.31 \\
\hline Trabajo en equipo & 7.34 & 3.16 & Puntualidad & 7.54 & 3.2 \\
\hline Puntualidad & 7.04 & 3.27 & Trabajo en equipo & 7.06 & 3.22 \\
\hline Amor al trabajo & 6.65 & 3.24 & Disciplina & 6.85 & 2.97 \\
\hline Disciplina & 6.41 & 3.19 & Amor al trabajo & 6.4 & 3.3 \\
\hline Confianza & 6.38 & 3.17 & Confianza & 6.39 & 2.88 \\
\hline Deseo de Superación & 5.97 & 3.07 & Deseo de Superación & 5.91 & 2.98 \\
\hline Honradez & 4.96 & 2.94 & Honradez & 4.98 & 2.89 \\
\hline Respeto a la persona & 4.11 & 3.25 & Responsabilidad & 3.78 & 2.25 \\
\hline Responsabilidad & 3.96 & 2.46 & Respeto a la persona & 3.07 & 2.65 \\
\hline
\end{tabular}




\section{CUADRO No 12}

\section{COMPARACIÓN DE VALORES ORGANIZACIONALES SEGÚN GÉNERO}

\begin{tabular}{|c|c|c|c|c|c|c|}
\hline GENERO & MA & ${ }_{6}^{\text {INO }}$ & & & $\mathbf{t}$ & Sign. \\
\hline Valores Organizacionales & $\mathbf{M}$ & D.S & $\mathbf{M}$ & D.S. & & \\
\hline Puntualidad & 7.04 & 3.27 & 7.54 & 3.20 & -8.96 &, $003 *$ \\
\hline Limpieza & 8.67 & 3.03 & 9.29 & 2.78 & -4.06 &, $000 *$ \\
\hline Calidad del Producto y Servicio & 7.53 & 3.47 & 7.8 & 3.31 & -0.54 &, 123 \\
\hline Deseo de Supervisión & 5.97 & 3.07 & 5.91 & 2.98 & 0.32 & ,746 \\
\hline Amor al Trabajo & 6.65 & 3.27 & 6.4 & 3.30 & 1.49 &, 136 \\
\hline Confianza & 6.38 & 3.17 & 6.39 & 2.88 & -0.12 & ,903 \\
\hline Responsabilidad & 3.96 & 2.46 & 3.78 & 2.25 & 1.48 &, 140 \\
\hline Respeto a la Persona & 4.11 & 3.24 & 3.07 & 2.64 & 6.75 &, $000 *$ \\
\hline Trabajo en Equipo & 7.34 & 3.16 & 7.01 & 3.23 & 1.67 & ,096 \\
\hline Honradez & 4.96 & 2.94 & 4.98 & 2.89 & -0.13 &, 152 \\
\hline Servicio al Cliente & 8.89 & 2.93 & 8.94 & 2.87 & -0.35 & ,730 \\
\hline Disciplina & 6.41 & 3.19 & 6.85 & 2.97 & -2.70 &, $007 *$ \\
\hline
\end{tabular}

* Significativo $p<0.5$

$G L: 1462$ 


\section{CUADRO No 13}

\section{CORRELACION ENTRE AUTOESTIMA Y VALORES ORGANIZACIONALES POR MUESTRA TOTAL Y POR UNIVERSITARIOS}

\begin{tabular}{|c|c|c|c|}
\hline $\begin{array}{c}\text { VALORES } \\
\text { ORGANIZACIONALES }\end{array}$ & $\begin{array}{c}\text { MUESTRA }(N \\
=1464) r\end{array}$ & $\begin{array}{c}\text { U.PUBL. } \\
=764) \quad \mathbf{N}\end{array}$ & $\begin{aligned} \text { U.PRIV. } & (\mathrm{N}= \\
700) & \mathbf{r}\end{aligned}$ \\
\hline \multirow[t]{2}{*}{ Puntualidad } & 0.043 & 0.072 & 0.001 \\
\hline & $\mathrm{p}=.098$ & $\mathrm{p}=.044^{*}$ & $\mathrm{p}=.970$ \\
\hline \multirow{2}{*}{ Limpieza } & 0.115 & 0.162 & 0.055 \\
\hline & $\mathrm{p}=.000^{*}$ & $\mathrm{p}=.000^{*}$ & $\mathrm{p}=.143$ \\
\hline \multirow[t]{2}{*}{ Calidad del Producto y Servicio } & 0.023 & -0.005 & 0.058 \\
\hline & $\mathrm{p}=.371$ & p 0.884 & $\mathrm{p}=.123$ \\
\hline \multirow[t]{2}{*}{ Deseo de superación } & 0.004 & 0.017 & -0.013 \\
\hline & $\mathrm{p}=.873$ & $\mathrm{p}=.631$ & $\mathrm{p}=.723$ \\
\hline \multirow[t]{2}{*}{ Amor al trabajo } & 0.002 & 0.026 & 0.015 \\
\hline & $\mathrm{p}=.400$ & $\mathrm{p}=.466$ & $\mathrm{p}=.703$ \\
\hline \multirow[t]{2}{*}{ Confianza } & -0.01 & 0.013 & -0.001 \\
\hline & $\mathrm{p}=.690$ & $\mathrm{p}=.713$ & $\mathrm{p}=.964$ \\
\hline \multirow[t]{2}{*}{ Responsabilidad } & -0.123 & -0.114 & -0.133 \\
\hline & $\mathrm{p}=.000^{*}$ & $\mathrm{p}=.002 *$ & $\mathrm{p}=.000^{*}$ \\
\hline \multirow[t]{2}{*}{ Respeto a la persona } & -0.052 & -0.041 & -0.06 \\
\hline & $\mathrm{p}=.043^{*}$ & $\mathrm{p}=.256$ & $\mathrm{p}=.109$ \\
\hline \multirow[t]{2}{*}{ Trabajo en equipo } & -0.38 & -0.074 & 0 \\
\hline & $\mathrm{p}=.140$ & $\mathrm{p}=.041^{*}$ & $\mathrm{p}=.994$ \\
\hline \multirow[t]{2}{*}{ Honradez } & -0.045 & -0.041 & 0.044 \\
\hline & $\mathrm{p}=.086$ & $\mathrm{p}=.262$ & $\mathrm{p}=.241$ \\
\hline \multirow[t]{2}{*}{ Servicio al cliente } & 0.031 & -0.011 & 0.054 \\
\hline & $\mathrm{p}=.232$ & $\mathrm{p}=.760$ & $\mathrm{p}=.148$ \\
\hline \multirow[t]{2}{*}{ Disciplina } & -0.006 & -0.031 & 0.023 \\
\hline & $\mathrm{p}=.831$ & $\mathrm{p}=.389$ & $\mathrm{p}=.528$ \\
\hline
\end{tabular}

* significativo $p<.05$

\section{CUADRO No 13}

COMPARACION DE LA AUTOESTIMA SEGÍUN GENERO

(MUESTRA TOTAL)

\begin{tabular}{|c|c|c|c|c|}
\hline & $\begin{array}{l}\text { Masculino } \\
\mathbf{N}^{\circ} 1236 \\
\text { D.S. }\end{array}$ & \begin{tabular}{cc}
\multicolumn{2}{c}{ Femenino } \\
$\mathrm{N}^{\circ} 228$ & $\mathrm{M}$ \\
& D.S.
\end{tabular} & $\mathbf{t}$ & Sign. \\
\hline Autoestima & $36.00 \quad 5.71$ & $36.23 \quad 5.05$ & -2.91 & $.004 *$ \\
\hline
\end{tabular}

*: Significativo $p<, 05$

$G L: 1462$ 
CUADRO No 31

NIVEL DE AUTOESTIMA POR UNIVERSITARIOS

\begin{tabular}{|c|c|c|c|c|}
\hline AUTOESTIMA & BAJO & MEDIO & ALTO & TOTAL \\
\hline & 271 & 268 & 225 & 764 \\
UNIVERSIDAD & 35.5 & 35.1 & 29.5 & 52.2 \\
PUBLICA & 52.4 & 55.6 & 48.4 & \\
& 18.5 & 18.3 & 15.4 & \\
\hline & 246 & 214 & 240 & 700 \\
UNIVERSIDAD & 35.1 & 30.6 & 34.3 & 47.8 \\
PRIVADA & 47.6 & 44.4 & 51.6 & \\
& 16.8 & 14.6 & 16.4 & \\
\hline TOTAL & 517 & 482 & 46.5 & 1464 \\
& 35.3 & 32.9 & 31.8 & 100.0 \\
\hline
\end{tabular}

*: Significativo a $p<, 05$

CUADRO $\mathbf{N}^{\circ} 32$

NIVEL DE AUTOESTIMA POR GENERO

\begin{tabular}{|c|c|c|c|c|}
\hline AUTOESTIMA & BAJO & MEDIO & ALTO & TOTAL \\
\hline & 287 & 220 & 229 & 736 \\
GENERO & 39.9 & 29.9 & 31.1 & 50.3 \\
MASCULINO & 55.5 & 45.6 & 49.2 & \\
& 19.6 & 15 & 15.6 & \\
\hline & 230 & 262 & 236 & 728 \\
GENERO & 31.6 & 36 & 32.4 & 49.7 \\
FEMENINO & 44.5 & 54.4 & 50.8 & \\
& 15.7 & 17.9 & 16.1 & \\
\hline TOTAL & 517 & 482 & 465 & 1464 \\
& 35.3 & 32.9 & 31.8 & 100 \\
\hline
\end{tabular}

*: Significativo a $p<, 05$ 
CUADRO No 33

NIVEL DE AUTOESTIMA POR ESTADO CIVIL

\begin{tabular}{|c|c|c|c|c|}
\hline AUTOESTIMA & BAJO & MEDIO & ALTO & TOTAL \\
\hline PROCEDENCIA & 35.4 & 401 & 397 & 1236 \\
LIMA & 84.7 & 83.2 & 85.4 & 84.4 \\
& 29.9 & 27.4 & 27.1 & \\
PROCEDENCIA & 34.6 & 31 & 68 & 228 \\
PROVINCIA & 15.3 & 16.8 & 14.6 & 15.6 \\
& 5.4 & 5.5 & 4.6 & \\
\hline TOTAL & 517 & 482 & 465 & 1464 \\
& 35.3 & 32.9 & 31.8 & 100 \\
\hline
\end{tabular}

* : Significativo a $p<, 05$

CUADRO No 34

NIVEL DE AUTOESTIMA POR GRUPOS DE EDAD

\begin{tabular}{|c|c|c|c|c|}
\hline AUTOESTIMA & BAJO & MEDIO & ALTO & TOTAL \\
\hline \multirow{2}{*}{ EDAD } & 234 & 234 & 200 & 668 \\
\multirow{2}{*}{$\mathbf{1 6 - 2 0}$} & 35.0 & 35.0 & 29.9 & 45.6 \\
& 45.3 & 48.5 & 43.0 & \\
& 16.0 & 16.0 & 13.7 & \\
\hline \multirow{2}{*}{ EDAD } & 28.0 & 248 & 265 & 796 \\
\multirow{2}{*}{$\mathbf{4 1}-\mathbf{4 8}$} & 35.6 & 31.2 & 33.3 & 54.4 \\
& 54.7 & 21.5 & 57.0 & \\
& 19.3 & 16.9 & 18.1 & \\
\hline TOTAL & 517 & 482 & 465 & 1464 \\
& 35.3 & 32.9 & 31.8 & 100.0 \\
\hline
\end{tabular}

* : Significativo a $p<, 05$ 


\section{CUADRO N $\mathbf{N}^{\circ} 35$}

NIVEL DE AUTOESTIMA POR ESTADO CIVIL

\begin{tabular}{|c|c|c|c|c|}
\hline AUTOESTIMA & BAJO & MEDIO & ALTO & TOTAL \\
\hline & 508 & 477 & 451 & 1436 \\
ESTADO CIVIL & 35.4 & 33.2 & 31.4 & 98.1 \\
SOLTERO & 98.3 & 99 & 97 & \\
& 34.7 & 32.6 & 30.8 & \\
ESTADO CIVIL & 32.1 & 17.9 & 50.0 & 1.9 \\
CASADO & 1.7 & 1.0 & 3.0 & \\
& 0.6 & 0.3 & 1.0 & \\
\hline TOTAL & 517 & 482 & 465 & 1464 \\
& 35.3 & 32.9 & 31.8 & 100 \\
\hline
\end{tabular}

*: Significativo a $p<, 05$ 